\title{
Transformations of an Auditory Temporal Code in the Medulla of a Sound-Producing Fish
}

\author{
James Kozloski and John D. Crawford \\ Graduate Group in Neuroscience and Department of Psychology, University of Pennsylvania, Philadelphia, Pennsylvania \\ 19104
}

The fish auditory system provides important insights into the evolution and mechanisms of vertebrate hearing. Fish have relatively simple auditory systems, without a cochlea for mechanical frequency analysis. However, as in all vertebrates, the primary auditory afferents of fish represent sounds as stimulusentrained spike trains. Thus, fish provide important models for studying how temporal spiking patterns are used in higher level neural computations. In this paper we demonstrate that one of the fundamental transformations of information in the auditory system of a sound-producing fish, Pollimyrus, takes place in the auditory medulla. We discovered a class of neurons in which evoked spiking patterns were relatively independent of the stimulus fine structure and appeared to reflect intrinsic properties of the neurons. These neurons generated sustained responses but were poorly phase-locked to tones compared

The transformations of acoustic information ascending the auditory system are fundamental in the study of auditory neural computation and the processing of communication sounds. Fish hold particular interest for the investigation of vertebrate hearing because of the relative simplicity of their ears. Fish have not evolved an elaborate structure for peripheral frequency analysis like the mammalian cochlea, and they have served as important model systems for studying auditory temporal computation (Fay, 1978a, 1994; Crawford, 1997b; Bodnar and Bass, 1999; Popper and Fay, 1999). The primary afferent neurons in the fish's auditory nerve, like those in other vertebrates, generate action potentials that are closely synchronized to features of sounds (Furukawa and Ishii, 1967; Fay, 1978b; Fay and Coombs, 1983; Moeng and Popper, 1984; Lu and Fay, 1996; Kozloski and Crawford, 1997, 1998a-c; McKibben and Bass, 1999; Suzuki and Crawford, 2000) and thus deliver a representation of the temporal structure of the stimulus to higher level circuits. In theory, the structure of these spike trains may be used to identify the frequency of a tone (Wever, 1949; Fay, 1970, 1978a, 1988; Marvit and Crawford, 2000) or to characterize more complex sounds (Fay and Passow, 1982; Fay, 1995). In this paper, we demonstrate

\footnotetext{
Received Nov. 19, 1999; revised Dec. 27, 1999; accepted Jan. 3, 2000.

This research was supported by the National Institutes of Health Grant R01 DC01252 and by the National Institute of Mental Health Predoctoral Fellowship Award PBN F31 MH11270 to J.K. We thank A. P. Cook, L. B. Fletcher, G. Garcia de Polavieja, P. Marvit, M. Nusbaum, J. C. Saunders, and A. Suzuki for their contributions to the preparation of this paper.

Correspondence should be addressed to Dr. John D. Crawford, University of Pennsylvania, 3815 Walnut Street, Philadelphia, PA 19104. E-mail: crawford@ psych.upenn.edu.

Dr. Kozloski's present address: Department of Biological Sciences, Columbia University, 1212 Amsterdam Avenue, New York, NY 10027.

Copyright (C) 2000 Society for Neuroscience $0270-6474 / 00 / 202400-09 \$ 15.00 / 0$
}

with the primary afferents. The interval histograms showed that spike timing was regular. However, in contrast to primary afferents, the mode of the interspike interval distribution was independent of the period of tonal stimuli. The tuning of the neurons was broad, with best sensitivity in the same spectral region where these animals concentrate energy in their communication sounds. The physiology of these neurons was similar to that of the chopper neurons known in the auditory brainstem of mammals. Our findings suggest that this medullary transformation, from phase-locked afferent input to chopper-like physiology, is basic to vertebrate auditory processing, even within lineages that have not evolved a cochlea.

Key words: auditory communication; chopper; computation; electric fish; hearing; temporal processing; neural transformation

that one of the major neural transformations of this primary temporal code occurs in the medulla of a sound-producing fish.

Pollimyrus adspersus is an African weakly-electric fish that has a well known repertoire of sounds used for courtship (grunts and moans) and specialization of the peripheral auditory system for sound pressure detection (Crawford, 1997a). Grunts are acoustic click trains (duration $\approx 250 \mathrm{msec}$ ) with an interclick interval of 18 msec that are produced in alternation with moans (duration $\approx$ $800 \mathrm{msec}$ ) by courting males. The moans are tonal, with sharp spectral peaks at 240 and $480 \mathrm{~Hz}$ (Crawford et al., 1997b). Recent research on the auditory nerve and medulla in Pollimyrus (Kozloski and Crawford, 1998a-c; Suzuki and Crawford, 2000) has shown that most primary afferents and first-order medullary neurons generate highly phase-locked, sustained responses to tones and click trains. However, in the course of these studies, we have discovered a distinct class of medullary neurons at the level of the secondary octaval nuclear complex in the medulla (SO) (McCormick and Hernandez, 1996; Kozloski and Crawford, 1998c). These neurons resemble the chopper neurons known throughout the mammalian auditory brainstem (Rhode, 1991). They produced sustained responses without phase locking to the stimulus fine structure, although they did have regular modes in their peristimulus time histograms. Thus, the physiology of these neurons represented a major transformation of auditory information in Pollimyrus.

Chopper responses have been described in a variety of vertebrates and across many nuclei in the brainstem. However, choppers have not been reported previously in the medulla of fish. Our findings show for the first time that this important neural transformation occurs early in auditory processing. Chopper-like responses, recorded in higher brain areas of fish (Lu and Fay, 1993, 
1995), may be created in the medulla and relayed to the midbrain and thalamus.

\section{MATERIALS AND METHODS}

The animals (Crawford, 1997a; Crawford et al., 1997a) and the methods used in our research (Crawford, 1993, 1997b; Kozloski and Crawford, 1998c) have been detailed previously. All of our animal protocols comply with The Principles of Animal Care published by the United States National Institutes of Health, and all were approved by the Institutional Animal Care and Use Committee of the University of Pennsylvania (Philadelphia, PA).

Pollimyrus adspersus (Mormyridae) were imported from Nigeria. Our analysis is based on 18 neurons recorded in 11 adult fish. The fish were immobilized by intramuscular injection of gallamine triethiodide (Flaxedil, $0.4 \mu \mathrm{g} / \mathrm{gm}$ of body weight) and placed in a water-filled acoustic tank within a sound-attenuating chamber. Physiological recordings were made while the fish was rigidly fixed $25 \mathrm{~mm}$ underwater in the tank, as described previously (Crawford, 1997b). Local anesthesia (lidocaine) was administered before the minor surgery required for microelectrode penetration of the medulla.

Acoustic signals were presented with an underwater speaker, and sound levels were expressed as decibels rms relative to $1 \mu \mathrm{Pa}$ for tones and decibels peak r.e. $1 \mu \mathrm{Pa}$ for clicks (subtract 100 for decibels relative to $1.0 \mathrm{dyne} / \mathrm{cm}^{2}$; subtract 26 for decibels relative to $20 \mu \mathrm{Pa}$ ). The maximum sound level used was $130 \mathrm{~dB}$, because this is approximately what the fish would encounter during short-range $(10 \mathrm{~cm})$ courtship interactions.

Extracellular recordings were made with metal-filled glass micropipettes [Indium electrodes (Dowben and Rose, 1953)] advanced into the brain with a Burleigh microdrive. After characterizing auditory neurons, we also checked for responses mediated by the lateral line mechanosensory system ( $30 \mathrm{~Hz}$ vibrating bead) and electrosensory system (electric dipole). None of the auditory neurons were activated by these stimuli. After physiology, standard neuroanatomical methods were used for tissue processing, histology, and anatomy [detailed in Kozloski and Crawford (1998c)].

Neurons were characterized by their responses to tones, clicks, and click trains. Tones were presented as short bursts $(100 \mathrm{msec})$, with 30 msec cosine rise/fall ramps and a repetition period of $1.15 \mathrm{sec}$. Prestimulus and poststimulus spikes were recorded for $100 \mathrm{msec}$ for each tone presentation. The clicks were $5 \mathrm{msec}$ pulses, with a flat amplitude spectrum in the $160 \mathrm{~Hz}$ to $5.0 \mathrm{kHz}$ band. Click trains were $400 \mathrm{msec}$ in duration, prestimulus and poststimulus spikes were recorded for $50 \mathrm{msec}$, and the train repetition period was $1.0 \mathrm{sec}$. The interclick interval (ICI) of the click trains was varied over a range from 10 to $80 \mathrm{msec}$ to check for interval selectivity. Clicks were usually presented at only a single sound level $(130 \mathrm{~dB})$, and no systematic study of click responses as a function of sound level was made.

All neurons were minimally characterized by their isolevel response function (ILRF) for tone frequency at $125 \mathrm{~dB}$ and by a spike rate versus sound level function [rate-level function (RLF)] and a peristimulus time histogram (PSTH with $50 \mu \mathrm{sec}$ resolution; $10 \mathrm{~dB}$ above threshold; $50-$ 100 trial repetitions) at the tone frequency evoking the maximum response [best frequency (BF)]. The ILRFs were constructed by measuring evoked spike rates while presenting a calibrated set of 22 log-spaced tone frequencies, ranging from $100 \mathrm{~Hz}$ to $3.3 \mathrm{kHz}$, in random sequence. Tuning was measured from the ILRF as the ratio of the BF to the bandwidth corresponding to the high and low frequency points at which the response dropped to $50 \%$ of maximum $\left(\mathrm{Q}_{50 \%}\right)$.

The RLFs were constructed by presenting BF tone bursts starting at 60 $\mathrm{dB}$ and increasing in $3 \mathrm{~dB}$ steps to $130 \mathrm{~dB}$. The threshold (in decibels) was measured from the RLF, and this was used as the best sensitivity (BS). The criterion for a threshold response was based on the spontaneous spike rate (SR) measured during the $100 \mathrm{msec}$ prestimulus periods: threshold spike rate $\geq$ mean SR $+2 \mathrm{SD}$. The dynamic range (in decibels) and the slope (spikes $\cdot$ second ${ }^{-1} \cdot$ decibel $^{-1}$ ) were also measured from the RLF. The dynamic range was defined as the range of stimulus sound levels (in decibels) over which a cell's firing rate was above criterion but was $<80 \%$ of its maximum evoked rate. The slope was computed by dividing the maximum evoked rate (Max-Criterion) by the corresponding dynamic range.

When neurons could be isolated for enough time, we constructed full response areas (RAs) by presenting the set of tones (random sequences), over a series of sound levels spaced at 3-5 dB. All tones were presented at a given sound level, a new level was selected at random, and tone presentations were repeated in a new random sequence. RAs spanned from below the neuron's BS (usually at $60 \mathrm{~dB}$ ) to $130 \mathrm{~dB}$. RAs were used to construct threshold tuning curves and then to measure the characteristic frequency, $\mathrm{BS}$, and $\mathrm{Q}_{10 \mathrm{~dB}}$. For each frequency, the threshold was taken as the first sound level that met our spike-rate criterion if the next higher sound level (usually $+3 \mathrm{~dB}$ ) also evoked a criterion response. The frequency with the lowest threshold (i.e., BS), and with a measured decrease in sensitivity of at least $10 \mathrm{~dB}$ for both higher and lower frequencies, was taken as the characteristic frequency (CF). BFs were also measured from the RA data sets, and this revealed that BF was correlated with CF $(r=0.69 ; p<0.02 ; \mathrm{df}=10)$ and thus a reasonable estimate of CF in choppers. The bandwidth (in Hertz) at $10 \mathrm{~dB}$ above BS was measured from the RA, and the ratio of CF to this bandwidth provided $\mathrm{Q}_{10 \mathrm{~dB}}$. A few neurons showed steadily decreasing thresholds as the frequency fell below $200 \mathrm{~Hz}$, and these neurons continued to do so to the lowest frequency presented $(100 \mathrm{~Hz}) ; \mathrm{BF}, \mathrm{BS}$, and $\mathrm{Q}_{10 \mathrm{~dB}}$ were not measured for these low-pass neurons.

The PSTH data were used to construct phase histograms and to make standard measurements of phase locking to the stimulus tones [synchronization coefficient or vector strength $r$ (Goldberg and Brown, 1969)]. The coefficients of variation (CVs) of the peristimulus interspike interval distributions were computed as a measure of the firing regularity $[\mathrm{CV}=$ $\mathrm{SD} /$ mean (Rees et al., 1997)]. Neurons that generated spikes at regular intervals had CVs near zero, and irregular interval distributions had larger CVs with no theoretical upper bound ( $\mathrm{CV}>1.0$ was not unusual). Some strongly phase-locked neurons have poor regularity, with large $\mathrm{CVs}$, because they do not fire a spike on every cycle of the stimulus waveform. A CV of 0.5 may be used as a criterion for regularity [regular if $\mathrm{CV} \leq 0.5$ (Young et al., 1988)].

The PSTH data were also used to measure spike-rate adaptation (SRA). Short-term SRA during single trials was measured from plots of instantaneous spike rate (i.e., reciprocal of interspike interval) as a function of time (i.e., peristimulus time). The slope of the best linear fit line was used as an estimate of SRA. For each neuron, the mean of six slopes, from the first six consecutive trials, was used as the estimate. Long-term SRA was examined by plotting the spike rate for each trial (i.e., spikes per $100 \mathrm{msec}$ tone) as a function of the time during the PST for the first 50 trials $(1 \mathrm{~min})$ and again using the slope of the best linear fit.

Means are presented with their SDs (mean \pm SD). Nonparametric Mann-Whitney $U$ tests were used to test for differences between distributions of those physiological measurements that were not normally distributed.

The data were collected during a study of the auditory nerve and medulla in which several hundred neurons were characterized (Kozloski and Crawford, 1997, 1998a-c; Suzuki and Crawford, 2000). The choppers were a distinct physiological class. We have made comparisons between these neurons and the primary-like neurons in the remaining medullary sample. The remaining medullary sample included primary afferents and primary-like medullary neurons. We have also provided some comparisons with the smaller sample of primary afferents that were identified by labeling. A detailed report on primary afferents and primary-like medullary neurons is in preparation.

\section{RESULTS}

We found a physiological type of medullary neuron that was distinct from primary afferents and other auditory neurons recorded in the medulla and that was similar to the choppers described in the brainstem of mammals. These auditory neurons were found deep in the medulla, 3000-3500 $\mu \mathrm{m}$ below the brain surface, at the level of the secondary octaval nuclear complex (Fig. 1). They were distinguished from other primary-like neurons in the medulla by the temporal structure of the spike trains generated in response to tones. Primary afferents and medullary neurons other than choppers produced highly phase-locked, sustained responses to tones (Kozloski and Crawford, 1998c; Suzuki and Crawford, 2000) (Fig. 2). Tone-evoked spike rates in primarylike neurons were often quite high (225.4 \pm 173.9 spikes/sec at $\mathrm{BF}$ ) because the spike rates were determined by the number of cycles in the stimulus. In contrast, chopper neurons produced sustained responses without phase locking to the stimulus fine structure and had significantly lower tone-evoked spike rates 


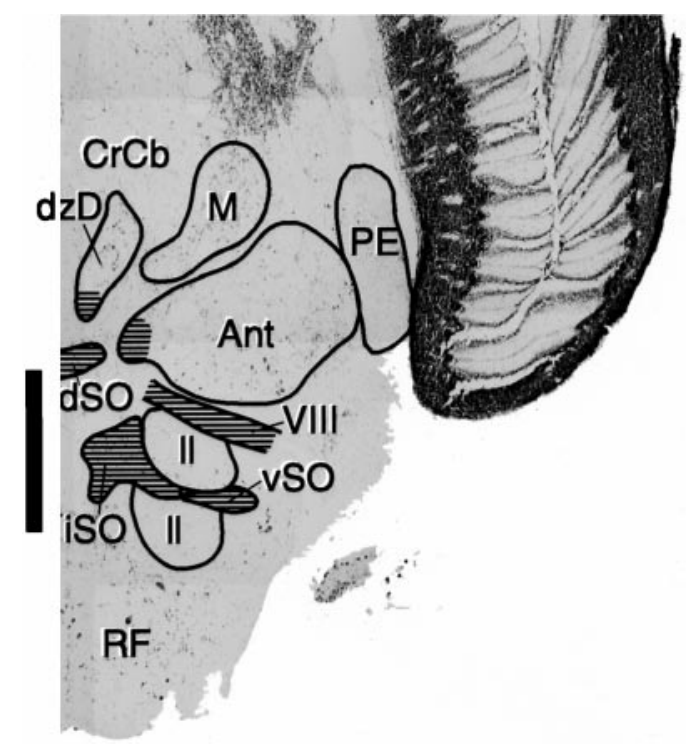

Figure 1. Transverse section through the auditory medulla of Pollimyrus, showing the area where choppers were recorded. Auditory regions are indicated by hatching. The vertical bar to the left of the $S O$ complex shows the range of depths, below the brain surface, at which recordings were made. [Additional details on anatomy are provided in Kozloski and Crawford (1998c).] Bar length, $500 \mu \mathrm{m}$. Ant, Anterior octaval nucleus; $C r C b$, crista cerebellaris; $d S O$, dorsal SO; $d z D$, dorsomedial zone of the descending nucleus; $i S O$, intermediate $\mathrm{SO}$; $l l$, lateral lemniscus; $M$, medial octaval nucleus; $P E$, preeminential toral nucleus; $R F$, reticular formation; $S O$, secondary octaval nucleus; $v S O$, ventral SO; VIII, eighth cranial nerve.

$(78.0 \pm 38.7$ spikes/sec; $U=874 ; p<0.0002)$. The peristimulus time histogram had a series of modes, a signature of the choppers that have been described in other systems.

Choppers in Pollimyrus were similar to the remaining medullary sample in their frequency selectivity. They responded most strongly to low frequencies (Fig. $3 A$ ), and there was no significant difference in the BF distributions of choppers and the remaining medullary sample $(U=1599 ; p=0.18)$. Choppers had slightly weaker frequency selectivity $\left(\mathrm{Q}_{50 \%}=1.98 \pm 1.94\right)$ than did the other medullary neurons $(2.59 \pm 2.33 ; U=1438 ; p<0.05)$. Eighty percent of choppers had a $\mathrm{Q}_{50 \%}$ below 2, compared with only $57 \%$ of the other medullary cells (Fig. $3 B$ ).

The choppers had simple, nonsaturating, rate-level functions (Fig. 4). The RLF slopes were significantly shallower for choppers $\left(2.2 \pm 2.3 \mathrm{spikes} \cdot \mathrm{sec}^{-1} \cdot \mathrm{dB}^{-1}\right)$ than for the remaining medullary sample $\left(8.7 \pm 5.7\right.$ spikes $\left.\cdot \mathrm{sec}^{-1} \cdot \mathrm{dB}^{-1} ; U=191 ; p<0.001\right)$. There was no significant difference in dynamic range between choppers $(24.06 \pm 15.3 \mathrm{~dB})$ and the other medullary neurons $(19.59 \pm 12.27 \mathrm{~dB} ; U=501 ; p=0.35)$. Choppers had low spontaneous rates $(13.9 \pm 10.4$ spikes/sec), but the distribution of spontaneous rates among the other medullary neurons was wide (55 \pm 88 spikes/sec), and the distributions were not significantly different $(U=3137 ; p=0.71)$.

We identified two subtypes of choppers by the characteristics of their spike trains, differences most conspicuously revealed by plotting responses to repeated tone presentations (raster plots). Stationary choppers $(n=12)$ had relatively high response rates and regular spike trains $(\mathrm{CV} \leq 0.5)$. They produced stereotyped sequences of interspike intervals, with a variable initiation latency, yielding characteristic patterns in the raster plots (Fig. 5). In contrast, nonstationary choppers $(n=6)$ had lower response rates and showed response
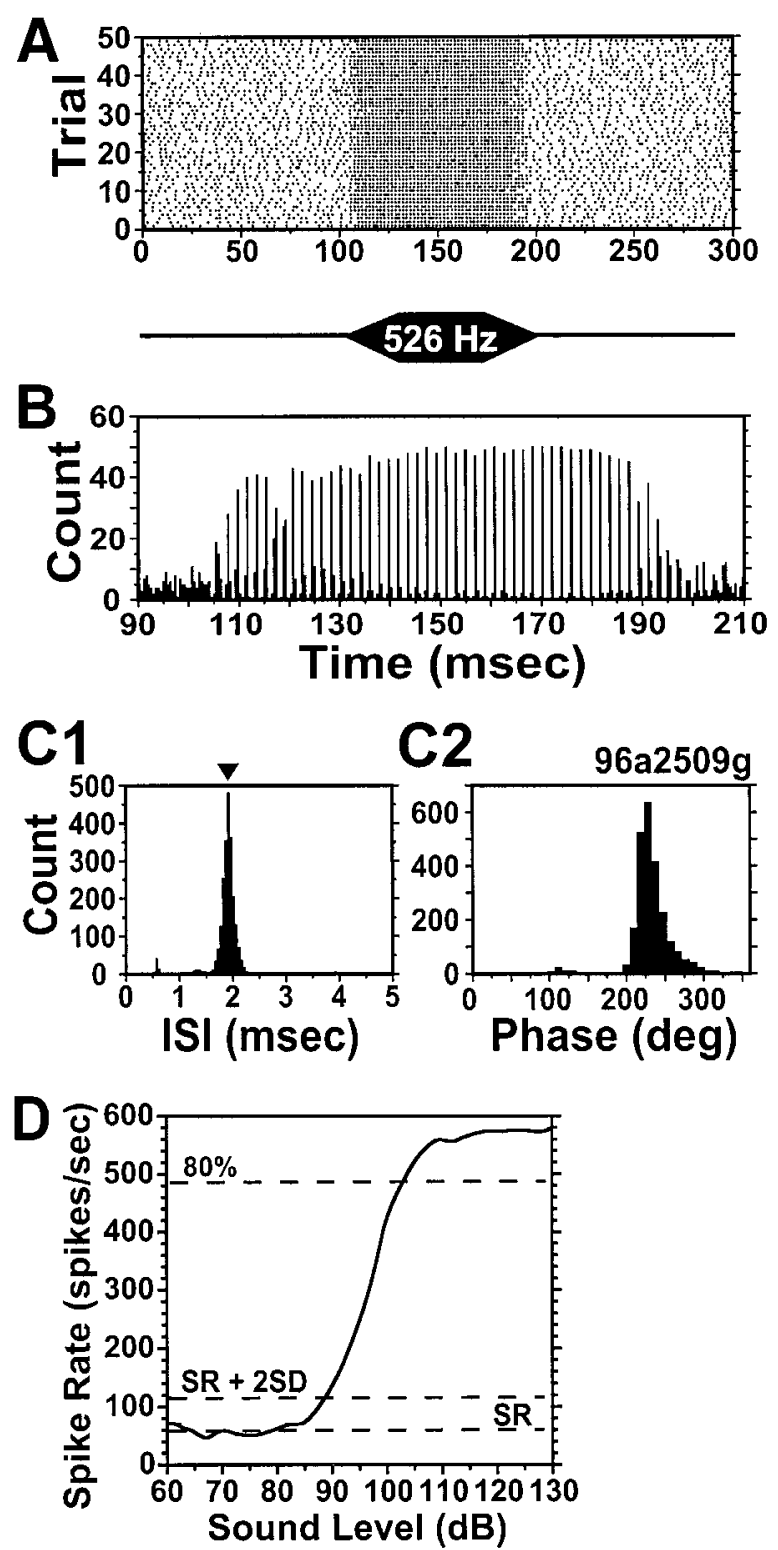

Figure 2. Primary-like medullary neuron. A, Raster display for 50 presentations of a $526 \mathrm{~Hz}$ tone. $B$, Peristimulus time histogram for data shown in $A$. $C$, Interspike interval histogram $(C 1)$ and phase histogram $(C 2)$ for peristimulus spikes. $D$, Rate-level function showing the SR and the criterion response (SR $+2 \mathrm{SD})$.

patterns that changed systematically with stimulus presentation number, over a time course of minutes (Fig. 6).

\section{Responses to tones}

Stationary choppers produced strong (93.3 $\pm 36.0 \mathrm{spikes} / \mathrm{sec})$, sustained responses to tones. Their interspike interval histograms (ISIH) were unimodal (Fig. 5C1), but they did not synchronize, and the position of the mode of the ISIH was primarily independent of the stimulus period. The ISI distributions were regular $(\mathrm{CV} \leq 0.5)$ for $83 \%$ of these neurons, and the coefficients of variation $(0.38 \pm 0.16)$ were similar to those in the primary afferents $(0.33 \pm 0.29)$. The phase histograms were nearly flat (Fig. 5C2), reflecting the poor synchronization $(r)$ of the spikes to the stimulus waveform $(0.19 \pm 0.17)$ compared with afferents $(0.88 \pm 0.13 ; U=0.00 ; p<0.0001)$. The stationary choppers 

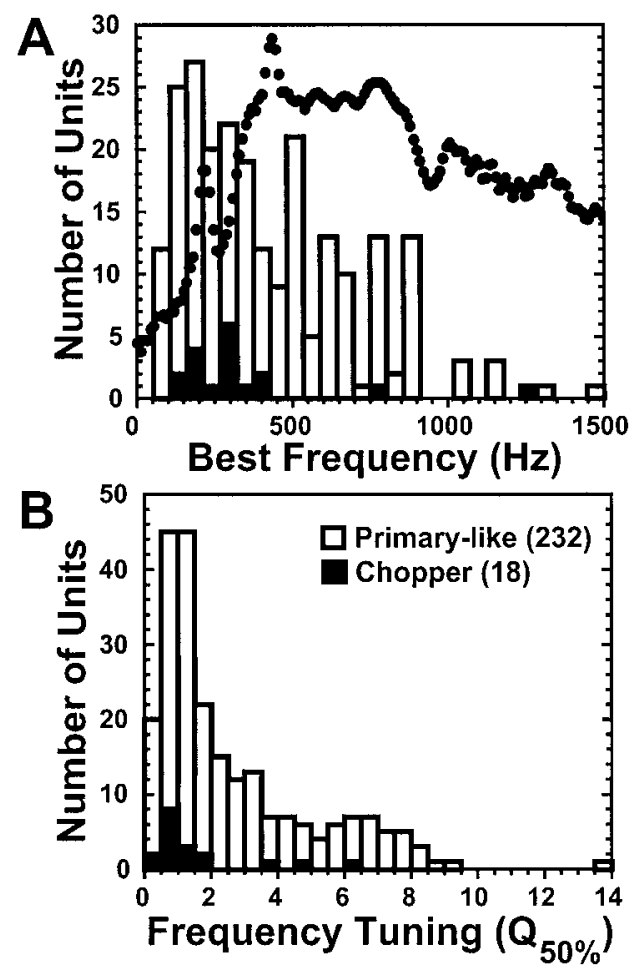

Figure 3. Summary of best frequency $(A)$ and frequency tuning $(B$; $\mathrm{Q}_{50 \%}$ ) for choppers (solid bars) and primary-like medullary neurons (open bars). The solid circles show the shape of the amplitude spectrum for a sequence of Pollimyrus sounds including grunts, moans, and a growl. The spectral peaks at 240 and $480 \mathrm{~Hz}$ are contributed by the tonal moan, and the grunts and growl add broad energy across the spectrum (see Crawford et al., 1997b).

exhibited substantial SRA over the course of the 100 msec stimulus (Fig. 5D), measured as the change in instantaneous spike rate as a function of time $(-197 \pm 149$ spikes/sec per $100 \mathrm{msec})$. Instantaneous spike rates at stimulus onset were occasionally as high as 300 spikes/sec but were generally lower than this (160 \pm $50 \mathrm{spikes} / \mathrm{sec})$. Average spike rates were stable over successive stimulus presentations, with no significant change over a full minute of stimulation (50 trials; repetition period $=1.15 \mathrm{sec}$; mean slope $=+4.5 \pm 23.4 \mathrm{spikes} \cdot \mathrm{sec}^{-1} \cdot \mathrm{min}^{-1} ; p>0.5$ for slope $\neq 0 ; t=0.66$; df $=11)$. These neurons were spontaneously active (15.32 \pm 11.57 spikes/sec) and often exhibited poststimulus suppression of this activity during the first $50 \mathrm{msec}$ of poststimulus time.

Stationary choppers generated successive spike trains that were quite similar to one another (i.e., stationary) but that varied in the onset latency. This feature of the spike trains yielded raster plots with a characteristic structure (Fig. $5 A$ ) and was apparent from cross-correlations between spike trains produced by neurons repeatedly stimulated with the same tone. For each pair of spike trains, we found the maximum correlation and the corresponding best temporal delay. The correlations $(r)$ were consistent $(0.4 \pm$ $0.03)$, but there was a great deal of variability in the best delays $($ mean SD $=26.04 \pm 9.46 \mathrm{msec}$ ).

The RAs of stationary choppers were broad, with the best sensitivity in the $200-400 \mathrm{~Hz}$ range (Fig. 5E). The stationary choppers produced similar responses in most positions within their RA. At the lowest frequencies $(\leq 122 \mathrm{~Hz})$, spikes became phase-locked to the stimulus, and this resulted in a shift in the ISI
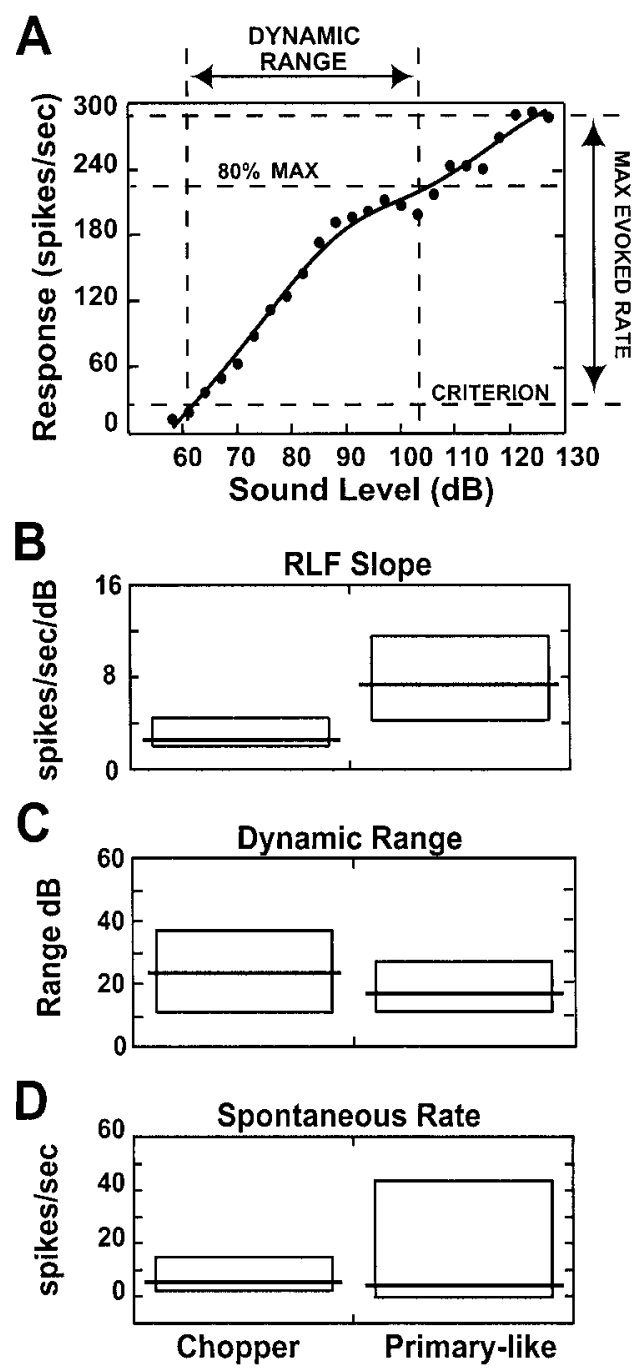

Figure 4. Rate-level functions for choppers $(A)$ had relatively shallow slopes $(B)$ and tended to have wide dynamic ranges $(C)$ compared with that of primary-like neurons (right side in $B, C$ ). Many choppers had low spontaneous rates compared with that of primary afferents $(D)$. Box plots $(B-D)$ show the median value (horizontal line) and the central $50 \%$ of the range of values (i.e., \pm 1 quartile). The difference between the RLF slopes of choppers and those of the neurons in the remaining sample was significant $(p<0.001)$, but there was no significant difference in dynamic range $(p>0.05)$. The $\operatorname{RLF}(A)$ is from a stationary chopper at $454 \mathrm{~Hz}$.

histogram toward the period of the stimulus. The ILRFs for stationary choppers were broad (mean $\mathrm{Q}_{50 \%}=1.54 \pm 1.57$ ), with response maxima at $\sim 400 \mathrm{~Hz}(385 \pm 274)$, and their RLFs were shallow and monotonic (Fig. $5 E$, inset).

Nonstationary choppers were similar to stationary choppers in many respects, but they showed conspicuous long-term changes in their temporal firing patterns (Fig. 6). The timing of the first pair of spikes in the response was relatively stable over trials. However, over successive tone presentations, the latencies of the later spikes in the response steadily increased, yielding a distinctive structure in the raster plots (Fig. 6A,C). This effect was most pronounced during the first minute of stimulation (50 trials). Like the stationary choppers, they exhibited poor synchronization to tones $(r=0.23 \pm$ $0.22)$, substantial SRA $(-121.03 \pm 64.05$ spikes/sec per $100 \mathrm{msec}$; $n=6$ ) during single-tone presentations, and no significant change in mean firing rate over the course of a minute of repeated stimulus 

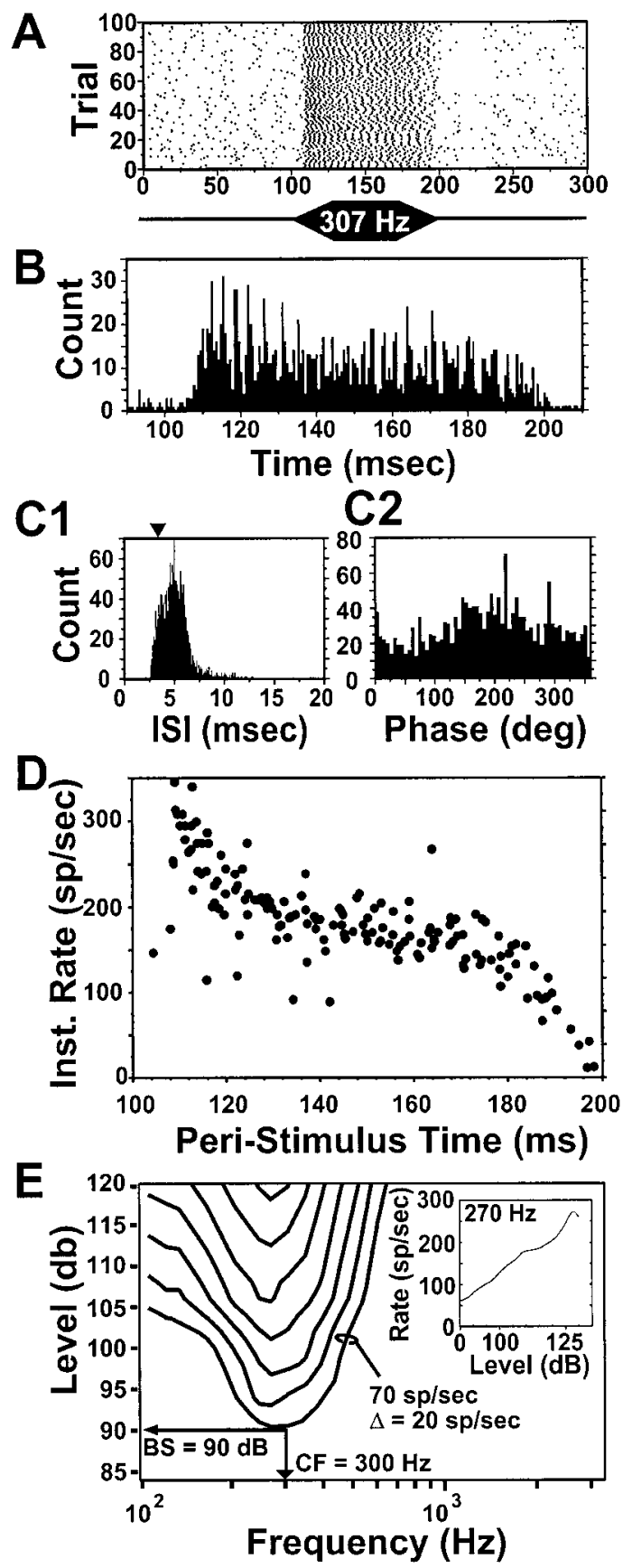

Figure 5. The temporal firing pattern $(A-D)$ and the response area $(E)$ and rate-level function $(E$, inset) for a stationary chopper stimulated with tones. Note the regular modes in the PSTH $(B)$ corresponding to the mode in the ISI histogram $(C 1)$ but the absence of phase locking $(C 2)$. The PSTH $(B)$ is plotted on an expanded time scale relative to the corresponding raster $(A)$ to illustrate better the temporal structure of the histogram. In the response area $(E)$, contour lines are separated by 20 spikes/sec. This neuron had a characteristic frequency of $300 \mathrm{~Hz}$, a best sensitivity of $90 \mathrm{~dB}$, and a $\mathrm{Q}_{10 \mathrm{~dB}}$ of 1 . Inst, Instantaneous; $s p$, spikes.

presentation (mean slope $=+1.494 \pm 8.37 \mathrm{spikes} \cdot \mathrm{sec}^{-1} \cdot \mathrm{min}^{-1}$; $p>0.5$ for slope $\neq 0 ; t=0.44 ; \mathrm{df}=5$ ). Instantaneous firing rates at stimulus onset were usually lower than those of stationary choppers (120 \pm 67 spikes/sec).

The nonstationary choppers had significantly lower driven rates $(47.53 \pm 23.83$ spikes/sec; $p=0.013 ; t=2.8 ; \mathrm{df}=16)$ and spontaneous rates $(1.07 \pm 1.36$ spikes $/ \mathrm{sec} ; p=0.009 ; t=2.96$;
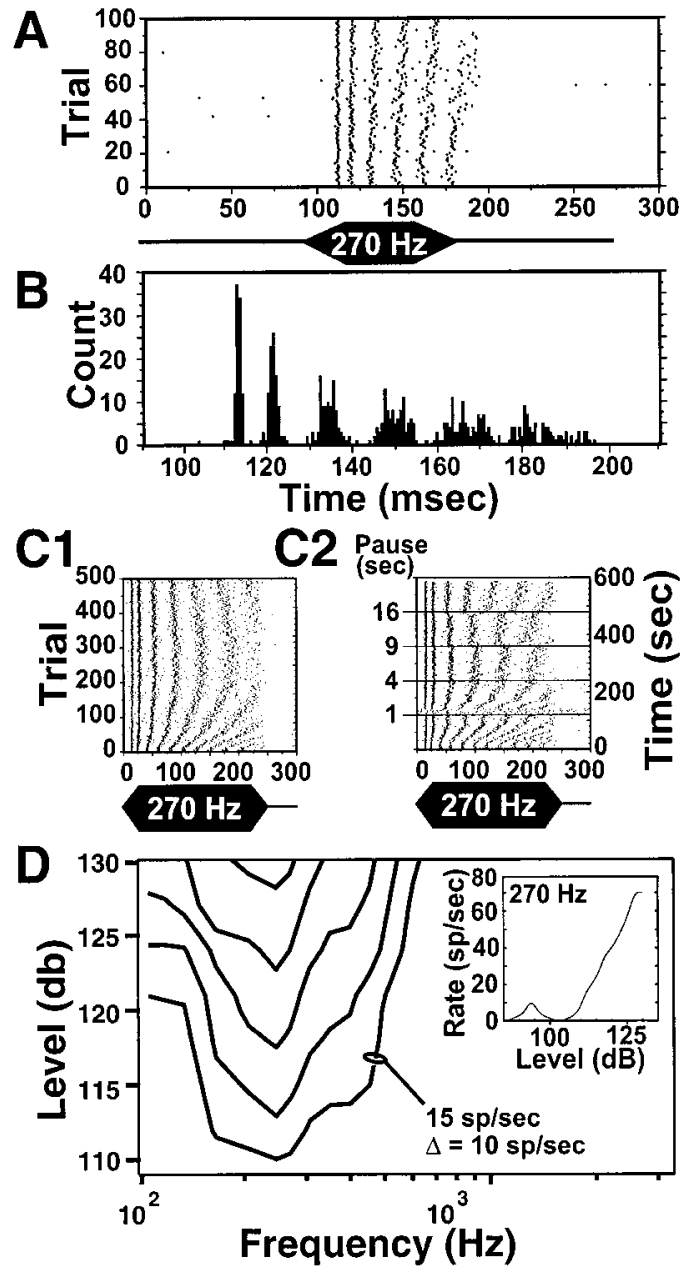

Figure 6. The temporal firing pattern $(A-C)$ and the response area $(D)$ and rate-level function $(D$, inset) for a nonstationary chopper stimulated with tones. $C 1$ shows a raster of 500 trials in which the temporal pattern of spikes underwent a systematic change. Pauses were introduced during the experiment illustrated in $C 2$. In the response area $(D)$, contour lines are separated by $10 \mathrm{spikes} / \mathrm{sec}$. This neuron had a characteristic frequency of $219 \mathrm{~Hz}$, a best sensitivity of $115 \mathrm{~dB}$, and a $\mathrm{Q}_{10 \mathrm{~dB}}$ of 0.6 .

$\mathrm{df}=16)$ than did the stationary choppers. Additionally, the ISI distributions for nonstationary choppers were more complicated with an initial mode corresponding to the first two spikes and other modes contributed by the dynamic portion of the response. The response areas of nonstationary choppers (Fig. 6D) were similar to those of stationary choppers, as were their isolevel response functions (Fig. $6 D$, inset; $\mathrm{Q}_{50 \%}=2.74 \pm 2.38$; $\mathrm{BF}=$ $385 \pm 390 \mathrm{~Hz}$ ). We were able to stimulate three of the nonstationary choppers at two to three different sound levels, and in no case did increases in sound level change the response from nonstationary to stationary.

To explore the long-term temporal dynamics of the nonstationary neurons, we increased the number of stimulus presentations and also introduced pauses into the stimulation regimen. We established a raster for responses to 500 tone presentations (repetition period $=1.14 \mathrm{sec}$ ) over a period of $9.5 \mathrm{~min}$ (Fig. $6 \mathrm{Cl}$ ). The most dramatic changes in spike latencies occurred within the first 100 trials $(2 \mathrm{~min})$, but systematic shifts in spike timing were evident throughout the test session. In some instances, the introduction of pauses (1-16 sec; 100 trial intervals) during a test 

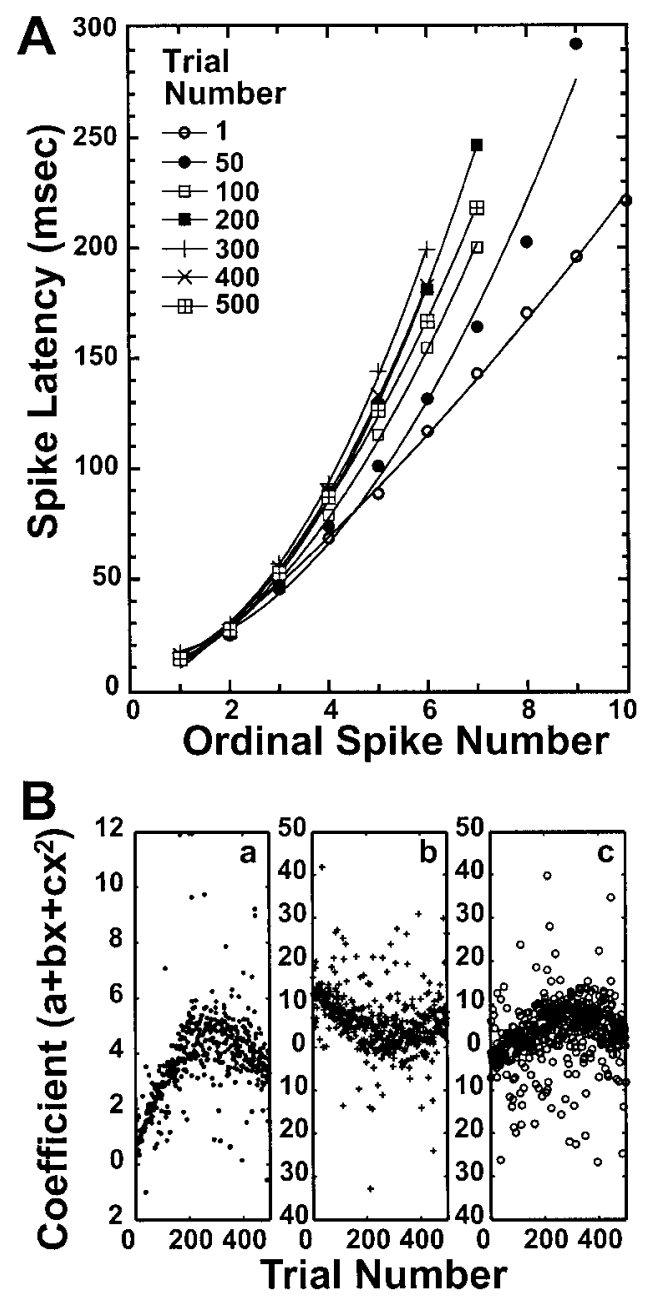

Figure 7. Polynomial fit of spike latencies for a nonstationary chopper (see Fig. $6 C$ ). $A$, For each of the seven example trials, spike latency was fit with a different polynomial. $B$, The coefficients resulting from fits for all 500 trials are shown; each trial was best fit with unique set of coefficients $(a, b, c)$.

session (600 trials) appeared to reset partially the temporal trajectory of the raster (Fig. 6C2). The influences of pauses were most pronounced during trials 1-200 when the spike pattern was changing most rapidly. The first two spikes of a spike train were relatively insensitive to the introduced pauses compared with later spikes.

The latency of each spike within the train varied systematically with trial number, as can be seen in the rasters (Fig. 6A,C). We examined the temporal trajectories of these spikes for all nonstationary choppers and found that they were closely fit (Corr $\geq$ 0.98 ) by second-order polynomial functions (Fig. $7 A$ ): latency $=$ $a+b x+c x^{2}$, where $x$ is the spike number within the train (Agmon and Connors, 1992). Each fit was evaluated by finding the scalar sum of observed (obs) and predicted (pred) latencies and normalizing:

$$
\text { Corr }=\frac{\sum_{i=1}^{N} L_{i}^{\mathrm{obs}} \cdot L_{i}^{\mathrm{pred}}}{\sqrt{\sum_{i=1}^{N}\left(L_{i}^{\mathrm{obs}}\right)^{2} \cdot \sum_{i=1}^{N}\left(L_{i}^{\mathrm{pred}}\right)^{2}}},
$$

where $N$ is the number of spikes in a trial and $L$ is the latency of the $i$ th spike.

The three coefficients ( $a, b$, and $c$ ) showed continuous, systematic changes over the course of 500 trials (Fig. 7B). They changed most rapidly during the first 100 trials when temporal response patterns underwent the most conspicuous changes. In the early trials, the trajectories were relatively simple with latency being essentially a linear function of spike number. As time progressed, the coefficient for the squared term $(c)$ increased steadily, reaching a maximum near trial 300 and then decreasing again out to trial 500. For each trial, the particular combination of coefficients that yielded the best fit for the whole trial also predicted the relatively trial-invariant latencies of the first two spikes (Fig. 7A), as observed in the physiology (Fig. 6). The polynomial equations provide a remarkably good mathematical description of changes in spike latency as a function of spike number within a trial and help to elucidate changes that occur from trial to trial. The closeness of these polynomial fits suggests that distinctive spiking properties of nonstationary choppers may be determined by at least three independent biophysical variables that vary continuously across trials.

One of the most important differences between choppers and other neurons encountered in the medulla was the relative independence of the interspike interval distribution and the stimulus frequency for choppers. The majority of the nonchopper medullary neurons showed phase-locked responses at all frequencies within the RA, and thus the mode of the ISI histogram showed a simple linear increase with tone period. This relationship clearly did not exist in the choppers (Fig. $8 A$ ). The average slope of the regression of mode ISI on stimulus period was not significantly different from zero $(-0.76 \pm 1.01 \mathrm{msec} / \mathrm{msec} ; p>0.05 ; t=2.24$; $\mathrm{df}=8)$. Similarly, an analysis of mode ISI and stimulus sound level revealed no systematic relationship (Fig. $8 B$; slope $=$ $-0.59 \pm 0.93 \mathrm{msec} / \mathrm{dB} ; p>0.05 ; t=1.91 ; \mathrm{df}=8)$. We have combined chopper types for these statistical analyses because of the small sample sizes of the two subtypes.

In terms of $\mathrm{CV}$ and $r$, choppers formed a distinct cluster that only overlapped a small part of the medullary sample (Fig. 9). The distribution of $r$ values for choppers differed significantly from that of the medullary sample $(U=277 ; p<0.001)$. None of the choppers had $r$ values $>0.78$. The $\mathrm{CV}$ distributions were not significantly different $(U=1843 ; p=0.61)$.

\section{Responses to clicks}

The two chopper subtypes had similar responses to clicks. Most (7 of 10) generated just one action potential after a single click and had a single postclick mode in the PSTH at $\sim 7 \mathrm{msec}$ (Fig. $10 \mathrm{~A})$. The mean response latency for choppers $(4.6 \pm 1.5 \mathrm{msec})$ was slightly longer than that for the afferents $(4.1 \pm 2.4 \mathrm{msec})$, but the difference was not significant $(p=0.54 ; t=0.63$; df $=21)$. Most of the choppers ( 8 of 10) generated single spikes locked $(r>$ $0.5)$ to the individual clicks of click trains (400 msec duration), as long as the interclick interval was of sufficient duration (ICI $\geq 18$ msec; Fig. 10B,C). The mean and SD for the synchronization coefficients and coefficients of variation were $0.68 \pm 0.26$ and $0.45 \pm 0.27$, respectively, for a click train with an $18 \mathrm{msec}$ ICI. The mean response to a $400 \mathrm{msec}$ click train $(\mathrm{ICI}=18 \mathrm{msec} ; 22$ clicks) was $32 \pm 12$ spikes/sec.

When the responses of choppers were examined as a function of interclick interval, 8 of 10 had a simple decrease in spike rate with increasing ICI, as expected from the declining total number of clicks in the fixed-duration trains (Fig. 10D). On a per-click basis, these neurons also showed a simple monotonic increase in 

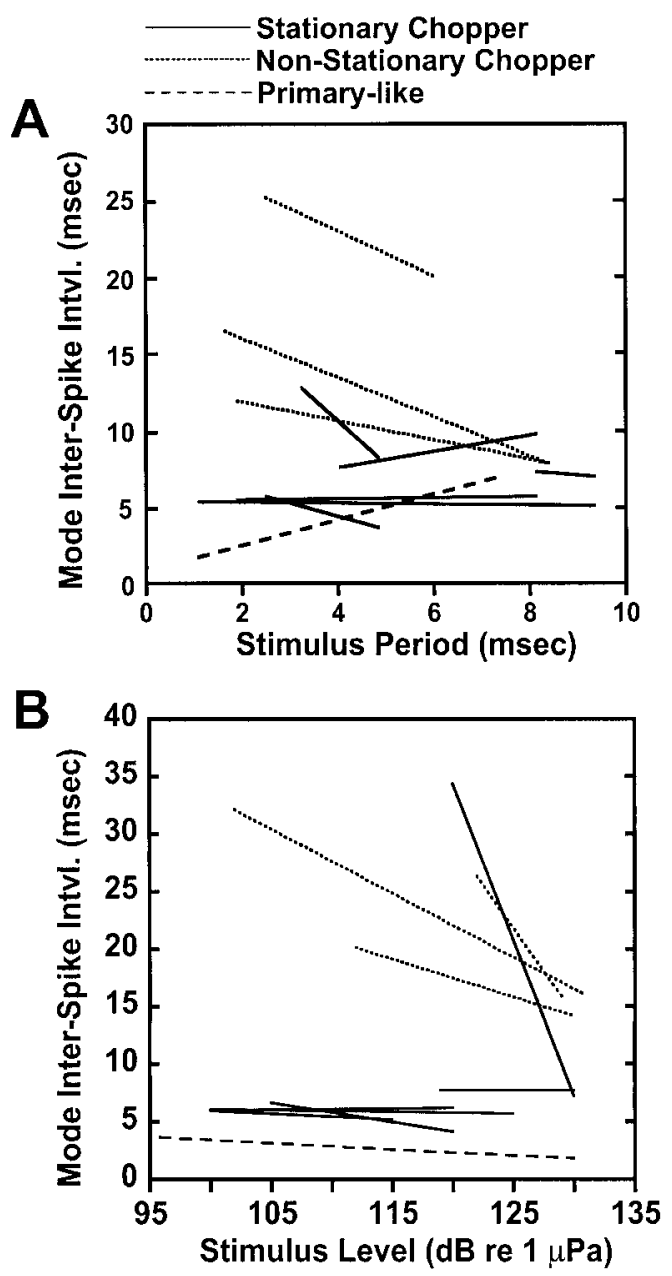

Figure 8 . The primary mode of the ISI distribution was not systematically related to either stimulus period $(A)$ or sound level $(B)$. Solid lines show the best linear fits for stationary choppers $(n=6)$, and dotted lines are for nonstationary choppers $(n=3)$. In contrast to choppers, primarylike neurons show a nearly perfectly linear relationship (slope $=1$ ) between stimulus period and the mode of the ISIH. The single dashed line in $A$ (slope $=1)$ and in $B$ (slope near zero) shows an example of a primary-like neuron for comparison. Intvl, Interval.

the probability of spiking as ICI was increased from a minimum of $10 \mathrm{msec}$ to an ICI of $80 \mathrm{msec}$ (Fig. 10E).

\section{DISCUSSION}

Chopper neurons are widespread among vertebrate taxa and must be fundamental in vertebrate auditory processing. Our data indicate that even among fish, in which time-based acoustic analysis may be particularly important (Fay, 1994), chopping is generated at the earliest stages of auditory computation. The choppers in Pollimyrus form a computational stream in which the information carried by afferent input about tone period in the form of ISIs undergoes a major transformation and is no longer explicitly represented in the output spike trains.

The Pollimyrus choppers had broad frequency-response functions with best sensitivity in the $100-500 \mathrm{~Hz}$ band, which matches the dominant energy in the sounds made by this species. While courting, males generate a two-part vocal display by alternating grunts and moans. The absence of synchronization to tones indicates that choppers would provide a poor temporal representation of the tonal waveform of the moan. However, there are

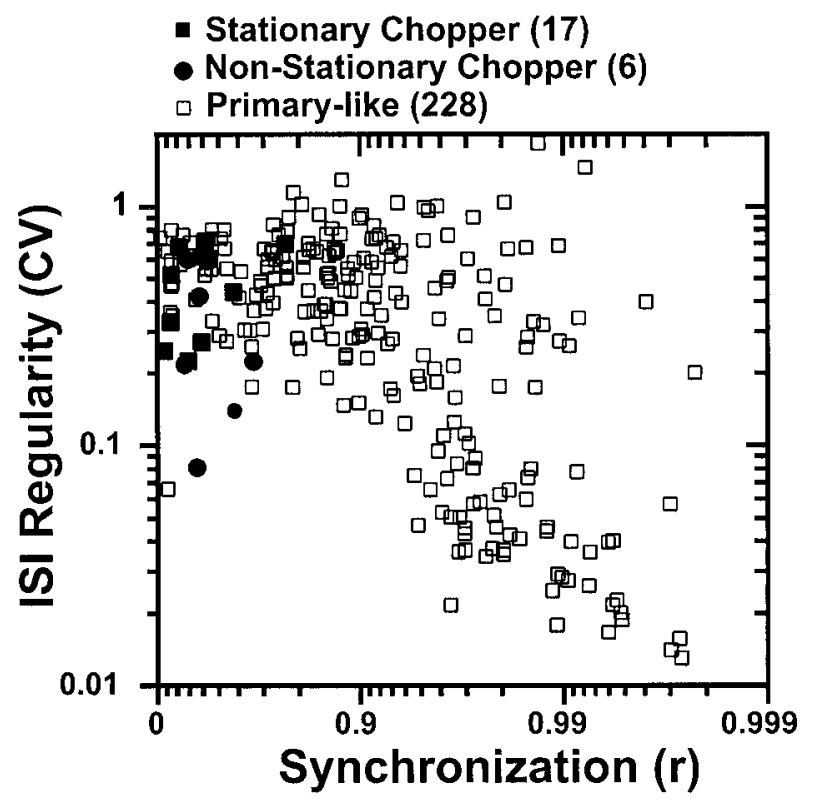

Figure 9. The CV and $r$ for choppers (solid symbols) and primary-like neurons (open squares) stimulated with tones. Note that choppers were relatively poorly phase-locked, with all $r$ values $<0.66$. The CVs of most choppers were $>0.20$. The $r$ and $\mathrm{CV}$ for choppers were $0.20 \pm 0.18$ and $0.34 \pm 0.16$, respectively. The $r$ and $\mathrm{CV}$ for other medullary neurons were $0.77 \pm 0.30$ and $0.39 \pm 0.35$, respectively. $r$ is plotted on a reversed log axis (see Joris et al., 1994). The compliment of the synchronization coefficient $(1-r)$ was plotted on a conventional, but left to right reversed, log scale, and the scale was then relabeled from 0 to 0.999 , left to right (i.e., instead of 1.0 to 0.001 , right to left ). This produced the plot of the original $r$ values on a reversed log axis.

three important ways in which these neurons appear to be suited to analysis of these natural sounds.

First, choppers may play a role in the analysis of moan amplitude. They showed a linear increase in spike rate with tone level and a wide dynamic range (Fig. 4). Several other studies of choppers have concluded that they are particularly suited to the coding of amplitude and amplitude modulations (Shofner and Dye, 1989; Sarbaz and Rees, 1996). Moans may be important in female choice (Crawford et al., 1997b). There are differences among individual males in their ability to generate moans that may predict mate quality. Moans are $\sim 800 \mathrm{msec}$ in duration, have a characteristic envelope with a gradual ramp up in intensity, and end relatively abruptly. Thus, the neural encoding of moan intensity by choppers could be important in communication.

Second, choppers provide a representation of the timing of clicks during trains, but only in the relatively long ICI range used in grunts (ICI $\geq 18 \mathrm{msec}$ ). Thus, choppers could provide the temporally coded input required for the creation of interval-selective responses in the midbrain and function in the analysis of grunts (Crawford, 1997b). However, most of the primary-like neurons also show strong synchronization to clicks, and over a wider range of ICIs than choppers. The synchrony at relatively long intervals by choppers may be important for certain analyses, but the primarylike neurons could also be used as input for interval analysis.

Third, the physiology of the nonstationary choppers is also intriguing in the context of moan analysis, because of the longterm changes of the response during presentations of tonal stimuli. Some individual males can produce a prolonged succession of moans, but others appear to fatigue and stop after producing only a few moans. Our results suggest that over the course of a natural 


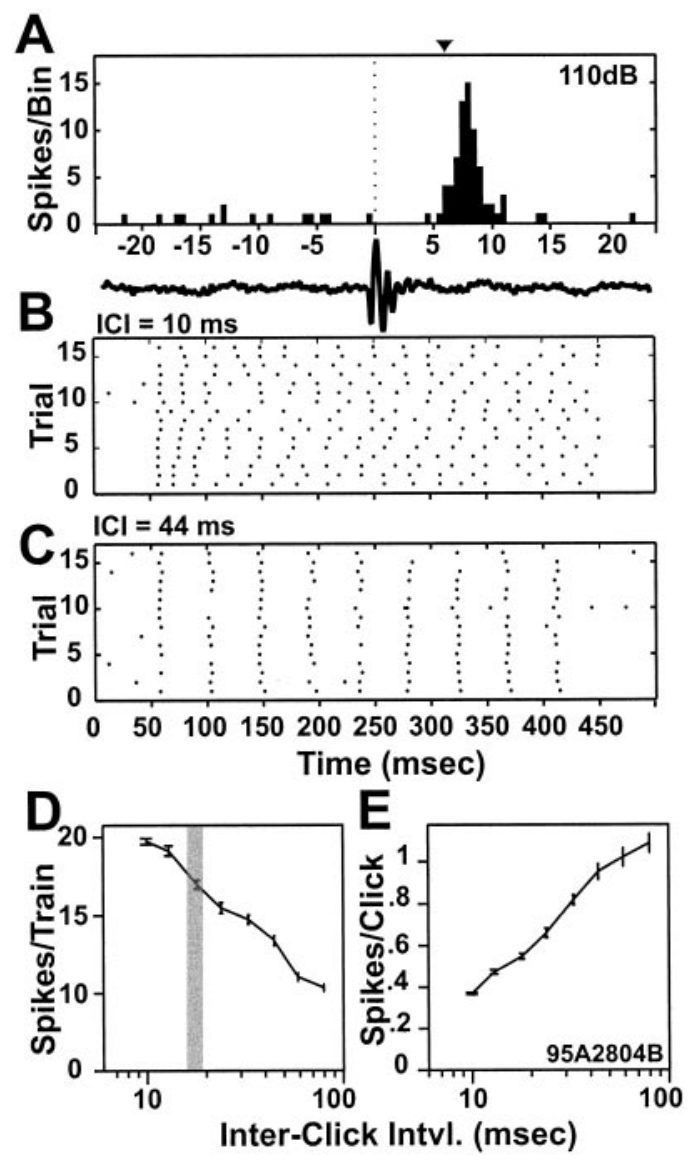

Figure 10. Responses of choppers to clicks. A, The distribution of responses after multiple presentations of a single click was usually unimodal with a mode at $\sim 7 \mathrm{msec}$ (arrowhead). $B, C$, During click trains, choppers gave sustained responses with weak synchronization at short interclick intervals $(B)$ and strong synchronization at longer ICIs $(C)$. $D$, Spike rates showed a simple monotonic dependence on ICI. $E$, Responses were normalized to the number of clicks per stimulus train, revealing that as ICI increases each click elicits more spikes. The width of the vertical gray bar in $D$ shows the center of the distribution of ICIs used in grunts (mean $\pm 1 \mathrm{SD})$.

courtship encounter $(\approx 20 \mathrm{sec})$, the output of nonstationary choppers would change substantially in response to a succession of moans (Fig. 6C) and potentially provide a readout of the duration of the sequence of moans. It is not yet clear how this putative temporal code for moan repetition might be analyzed.

Choppers have been described in the olivary complex of other vertebrates (Harnischfeger et al., 1985; Finlayson and Adam, 1997), an auditory region that is critical in binaural processing of spatial cues. Although comparatively little is currently known about the neural computations involved in spatial hearing in fish (Fay, 1984; Rogers et al., 1988; Edds-Walton and Fay, 1998; Lu et al., 1998), choppers in the SO of Pollimyrus could also be involved in the spatial processing of acoustic information [discussed further in Kozloski and Crawford (1998c)].

Choppers resembling the stationary choppers of Pollimyrus have been reported in goldfish and a number of other vertebrates. In a study of the goldfish auditory midbrain (Lu and Fay, 1993), a small subset of nonphase-locked neurons were similar to the stationary choppers in the medulla of Pollimyrus. The goldfish neurons gave sustained responses and produced regular interspike interval distributions that were independent of the stimulus period. Lu and Fay (1993) used sharp micropipettes, good for isolating input fibers, and the chopper-like physiology they recorded in the midbrain might have originated in the goldfish medulla, as in Pollimyrus. Lu and Fay (1995) also describe several chopper-like types in the auditory thalamus of goldfish, but these appear less similar to the stationary choppers in Pollimyrus and probably reflect additional physiological processing, or a de novo computation from nonchopping inputs.

The physiology and anatomy of choppers have been most thoroughly studied in mammals (Young et al., 1988; Rhode and Greenberg, 1992; Rees et al., 1997). The stationary choppers in Pollimyrus are similar in many respects to the sustained chopper type $\left(\mathrm{C}_{\mathrm{s}}\right)$ found in the mammalian brainstem. In both vertebrates, the response to tones persists throughout the stimulus (sustained), the PSTH reveals a series of modes (chopping pattern), and the ISIH is usually unimodal, with a low CV and a mode that is independent of stimulus period. They both have relatively high driven rates and wide dynamic ranges and show some phase locking if the stimulus frequency is low enough.

Sustained choppers in mammals show suppression of spike rate to tones presented outside of the excitatory range (side-band suppression), but we have not observed this in Pollimyrus. Additionally, the excitatory receptive fields of mammalian choppers are at higher frequencies $(\mathrm{BF} \geq 1 \mathrm{kHz})$ than are those of Pollimyrus. In the mammalian cochlear nucleus, sustained choppers are neurons that have a stellate morphology (Rhode et al., 1983), with relatively long dendrites with few branches, and that receive large numbers of excitatory and inhibitory synaptic inputs (Smith and Rhode, 1989). We do not know yet whether the same morphological correlates exist in Pollimyrus.

In contrast to the stationary choppers, we know of no other neurons like the Pollimyrus nonstationary choppers reported from other in vivo studies of auditory systems. However, examples resembling Pollimyrus nonstationary choppers have been reported in slice preparations. Sustained depolarizing current injections in fusiform auditory neurons of the rat lateral superior olivary complex produced spiking patterns that were similar to that of the Pollimyrus nonstationary choppers. They had an initial short latency pair of spikes followed by a dynamic spike train, regular ISI distributions, and multimodal PSTHs (Adam et al., 1999). Pyramidal neurons in the visual cortex of rats (Mainen and Sejnowski, 1995) and mice (J. Kozloski and R. Yuste, unpublished observations) stimulated with regular DC current pulses also produced firing patterns similar to those evoked by sound in the Pollimyrus nonstationary choppers. These studies suggest that the distinctive firing patterns of nonstationary choppers reflect intrinsic membrane properties that occur in many neuronal types and that nonstationary choppers receive multiple excitatory synaptic inputs that together yield sustained depolarization during auditory stimulation (Oertel et al., 1988).

The chopper neurons in Pollimyrus appear to correspond to one branch of a bifurcating input stream that is suited to intensity analysis. A second parallel time-coding pathway seems to depend on auditory neurons in the descending nucleus of the medulla (Kozloski and Crawford, 1998c). Diverging primary afferent input and the formation of parallel time and intensity analysis streams are well known in other vertebrate sensory systems, including the electrosensory system of fish (Heiligenberg, 1991) and the auditory systems of birds (Konishi, 1993) and mammals (Harnischfeger et al., 1985; Suga, 1990). Discovering the close similarities between particular physiological types of neurons in Pollimyrus and mammals, such as choppers, increases our confi- 
dence that continued analysis will yield important insight into auditory processing in all vertebrates.

\section{REFERENCES}

Adam TJ, Schwarz DW, Finlayson PG (1999) Firing properties of chopper and delay neurons in the lateral superior olive of the rat. Exp Brain Res 124:489-502.

Agmon A, Connors BW (1992) Correlation between intrinsic firing patterns and thalamocortical synaptic responses of neurons in mouse barrel cortex. J Neurosci 12:319-329.

Bodnar DA, Bass AH (1999) Midbrain combinatorial code for temporal and spectral information in concurrent acoustic signals. J Neurophysiol 81:552-563.

Crawford JD (1993) Central auditory neurophysiology of a sound- producing mormyrid fish: the mesencephalon of Pollimyrus isidori. J Comp Physiol [A] 172:1-14.

Crawford JD (1997a) Hearing and acoustic communication in the mormyrid electric fishes. Mar Fresh Behav Physiol 29:1-21.

Crawford JD (1997b) Feature-detecting auditory neurons in the brain of a sound-producing fish. J Comp Physiol [A] 180:439-450.

Crawford JD, Jacobe P, Benech V (1997a) Field studies of a strongly acoustic fish in West Africa: reproductive ecology and acoustic behavior of Pollimyrus isidori, Mormyridae. Behaviour 134:677-725.

Crawford JD, Cook AP, Heberlein AS (1997b) Bioacoustic behavior of African fishes (Mormyridae): potential cues for species and individual recognition in Pollimyrus. J Acoust Soc Am 102:1200-1212.

Dowben RM, Rose JE (1953) A metal-filled electrode. Science 118:22.

Edds-Walton PL, Fay RR (1998) Directional auditory responses in the descending octaval nucleus of the toadfish (Opsanus tau). Biol Bull 195:191-192.

Fay RR (1970) Auditory frequency discrimination in the goldfish (Carassius auratus). J Comp Physiol Psychol 73:175-180.

Fay RR (1978a) Phase-locking in goldfish saccular nerve fibers accounts for frequency discrimination capacities. Nature 275:320-322.

Fay RR (1978b) Coding of information in single auditory-nerve fibers of the goldfish. J Acoust Soc Am 63:136-146.

Fay RR (1984) The goldfish ear codes the axis of acoustic particle motion in three dimensions. Science 225:951-954.

Fay RR (1988) Hearing in vertebrates: a psychophysics databook. Winnetka, IL: Hill-Fay.

Fay RR (1994) Perception of temporal acoustic patterns by the goldfish (Carassius auratus). Hear Res 76:158-172.

Fay RR (1995) Perception of spectrally and temporally complex sounds by the goldfish (Carassius auratus). Hear Res 89:146-154.

Fay RR, Coombs S (1983) Neural mechanisms in sound detection and temporal summation. Hear Res 10:69-92.

Fay RR, Passow B (1982) Temporal discrimination in the goldfish. J Acoust Soc Am 72:753-760.

Finlayson P, Adam TJ (1997) Excitatory and inhibitory response adaptation in the superior olive complex affects binaural acoustic processing. Hear Res 103:1-18.

Furukawa T, Ishii Y (1967) Neuorophysiological studies on hearing in goldfish. J Neurophysiol 30:1377-1403.

Goldberg JM, Brown PB (1969) Response of binaural neurons of dog superior olivary complex to dichotic tonal stimuli: some physiological mechanisms of sound localization. J Neurophysiol 32:613-636.

Harnischfeger G, Neuweiler G, Schlegel P (1985) Interaural time and intensity coding in superior olivary complex and inferior colliculus of the echolocating bat Molossus ater. J Neurophysiol 53:89-109.

Heiligenberg W (1991) Neural nets in electric fish. Cambridge, MA: MIT.

Joris PX, Carney LH, Smith PH, Yin TCT (1994) Enhancement of neural synchronization in the anteroventral cochlear nucleus. I. Responses to tones at the characteristic frequency. J Neurophysiol 71:1022-1036.

Konishi M (1993) Neuroethology of sound localization in the owl. J Comp Physiol [A] 173:3-7.

Kozloski J, Crawford JD (1997) Physiology of primary afferents and first order auditory neurons in the medulla of a sonic fish: Pollimyrus adspersus. Assoc Res Otolaryngol Abstr 1997:566.

Kozloski J, Crawford JD (1998a) Rate coding of sound features in second and third order auditory nuclei is computed from temporally structured first order spike trains. Soc Neurosci Abstr 24:186.

Kozloski J, Crawford JD (1998b) A model of computation through con- vergence and multiple spike initiation zones predicts the enhanced temporal representation of auditory signals in the medulla of Pollimyrus. Int Soc Neuroethology Abstr 1998:66.

Kozloski J, Crawford JD (1998c) Functional neuroanatomy of auditory pathways in the sound producing fish Pollimyrus. J Comp Neurol 401:227-252.

Lu Z, Fay R (1996) Two-tone interaction in primary afferents and midbrain neurons of the goldfish, Carassius auratus. Audit Neurosci 2:257-273.

Lu Z, Fay RR (1993) Acoustic response properties of single units in the torus semicircularis of the goldfish, Carassius auratus. J Comp Physiol [A] 173:33-48.

Lu Z, Fay RR (1995) Acoustic response properties of single neurons in the central posterior nucleus of the thalamus of the goldfish, Carassius auratus. J Comp Physiol [A] 176:747-760.

Lu Z, Song J, Popper AN (1998) Encoding of acoustic directional information by saccular afferents of the sleeper goby, Dormitator latifrons. J Comp Physiol [A] 182:805-815.

Mainen ZF, Sejnowski TJ (1995) Reliability of spike timing in neocortical neurons. Science 268:1503-1506.

Marvit P, Crawford JD (2000) Auditory discrimination in a soundproducing fish, Pollimyrus (Mormyridae). Assoc Res Otolaryngol Abstr 2000:977.

McCormick CA, Hernandez DV (1996) Connections of octaval and lateral line nuclei of the medulla in the goldfish, including the cytoarchitecture of the secondary octaval population in goldfish and catfish. Brain Behav Evol 47:113-137.

McKibben JR, Bass AH (1999) Peripheral encoding of behaviorally relevant acoustic signals in a vocal fish: single tones. J Comp Physiol [A] 184:563-576.

Moeng RS, Popper AN (1984) Auditory response of saccular neurons of the catfish, Ictalurus punctatus. J Comp Physiol [A] 155:615-624.

Oertel D, Wu AH, Hirsch JA (1988) Electrical characteristics of cells and neuronal circuitry in the cochlear nuclei studied with intracellular recordings from brain slices. In: Auditory function: neurobiological basis of hearing (Edelman GM, Gall WE, Cowan WM, eds), pp 313-336. New York: Wiley.

Popper AN, Fay RR (1999) The auditory periphery in fishes. In: Comparative hearing: fish and amphibians (Fay RR, Popper AN, eds), pp 43-100. New York: Springer.

Rees A, Sarbaz A, Malmierca MS, Le Beau FE (1997) Regularity of firing of neurons in the inferior colliculus. J Neurophysiol 77:2945-2965.

Rhode WS (1991) Physiological-morphological properties of the cochlear nucleus. In: Neurobiology of hearing: the central auditory systems (Altschuler RA, Clopton BM, Bobbin BM, Hoffman DW, eds), pp 47-77. New York: Raven.

Rhode WS, Greenberg S (1992) Physiology of the cochlear nuclei. In: The mammalian auditory pathway: neurophysiology (Popper AN, Fay RR, eds), pp 94-152. New York: Springer.

Rhode WS, Oertel D, Smith PH (1983) Physiological response properties of cells labeled intracellularly with horseradish peroxidase in cat ventral cochlear nucleus. J Comp Neurol 213:448-463.

Rogers PH, Popper AN, Hastings MC, Saidel WM (1988) Processing of acoustic signals in the auditory system of bony fish. J Acoust Soc Am 83:338-349.

Sarbaz A, Rees A (1996) Amplitude modulation encoding and regularly firing neurons in the inferior colliculus. Br J Audiol 30:105-106.

Shofner WP, Dye RH (1989) Statistical and receiver operating characteristic analysis of empirical spike-count distributions: quantifying the ability of cochlear nucleus units to signal intensity changes. J Acoust Soc Am 86:2172-2184.

Smith PH, Rhode WS (1989) Structural and functional properties distinguish two types of multipolar cells in the ventral cochlear nucleus. J Comp Neurol 282:595-616.

Suga N (1990) Cortical computational maps for auditory imaging. Neural Networks 3:3-21.

Suzuki A, Crawford JD (2000) The primary auditory input to the brain of sound-producing mormyrid fishes. Assoc Res Otolaryngol Abstr 2000:976.

Wever E (1949) Theory of hearing. New York: Wiley.

Young ED, Robert JM, Shofner WP (1988) Regularity and latency of units in ventral cochlear nucleus: implications for unit classification and generation of response properties. J Neurophysiol 60:1-29. 